\section{Regards sur l'économie allemande}

Bulletin économique du CIRAC

$104 \mid 2012$

Varia

\title{
Niveau des qualifications et distribution des revenus
}

\section{Matthias Bittorf et Alexander Klein}

Traducteur : Isabelle Bourgeois

\section{OpenEdition \\ Journals}

Édition électronique

URL : http://journals.openedition.org/rea/4390

DOI : $10.4000 /$ rea.4390

ISBN : 978-2-8218-1286-4

ISSN : 1965-0787

Éditeur

CIRAC

Édition imprimée

Date de publication : 13 avril 2012

Pagination : 15-22

ISSN : 1156-8992

\section{Référence électronique}

Matthias Bittorf et Alexander Klein, « Niveau des qualifications et distribution des revenus », Regards sur l'économie allemande [En ligne], 104 | avril 2012, mis en ligne le 01 avril 2014, consulté le 19 avril 2019. URL : http://journals.openedition.org/rea/4390 ; DOI : 10.4000/rea.4390 


\section{Niveau des qualifications et distribution des revenus}

\author{
Matthias Bittorf, Alexander Klein
}

La question de la disparité des revenus (re)surgit à intervalles réguliers dans l'espace public allemand. Ces dernières années, elle prend la forme de controverses sur l'émergence d'une nouvelle "classe inférieure ", voire d'un nouveau " précariat " (Unterschicht), et se focalise depuis sur le risque d'une spirale d'exclusion (Köcher, 2011), ou encore sur l'adoption de salaires minima légaux en réponse à ces évolutions. Toutes ces questions sont volontiers et abondamment relatées actuellement par la presse en France où elles servent aussi à nourrir la polémique sur ce "modèle allemand" qu'il conviendrait d'imiter. Or ces débats étant rarement factuels, seule une analyse détaillée des données statistiques permet de replacer les divers constats dans leur contexte et ainsi d'apporter la sérénité indispensable pour aborder la problématique dans sa complexité et comprendre le rôle déterminant que joue le niveau des qualifications dans l'inégalité des revenus.

La ressource clé qui permet à la plupart des citoyens d'atteindre et de conserver le standard de vie désiré est le revenu issu du travail. Sa répartition au sein de l'ensemble des actifs est considérée généralement comme le facteur déterminant pour considérer l'inégalité sous l'angle économique. Pour l'approche macro-économique, un autre facteur important est la répartition du patrimoine ; mais nous ne le prendrons pas en considération ici étant donné que les statistiques ne l'éclairent que très partiellement et qu'il n'y a notamment pas de données récentes quant à son évolution. Pour évaluer si la répartition des revenus est raisonnable ou équitable, il convient de peser avantages et désavantages en considérant deux aspects principalement. D'un côté, une certaine inégalité dans la distribution des revenus reflète celle de la performance économique des actifs ; elle est donc l'expression de la juste rétribution de la qualité du travail. De l'autre, si on veut garantir la participation de chacun à la vie économique et culturelle et ainsi assurer la cohésion sociale, les inégalités de revenus ne sont acceptables que jusqu'à une certaine limite.

Or c'est le niveau de formation qui est le facteur clé pour une participation pleine et entière à l'activité. Et, en Allemagne, tout particulièrement le diplôme sanctionnant l'achèvement d'une formation professionnelle initiale qui est le sésame de l'intégration professionnelle, et la référence en ce qui concerne le revenu attendu de même que l'ascension professionnelle espérée. Dans un pays aux salaires élevés comme l'Allemagne, où la demande de travail faiblement qualifié se réduit comme peau de chagrin alors que la demande de travail hautement qualifié ne cesse d'augmenter (cf. Franz, 2009 ; voir aussi dans ce numéro la contribution de Spitznagel/Wanger), le niveau de qualification détenu est particulièrement déterminant. En outre, plus il est élevé, et plus la productivité des actifs s'accroît...

\section{Hausse des inégalités dans le revenu net des ménages}

Globalement, l'inégalité des revenus a augmenté en Allemagne depuis les années 1990. Selon une récente étude publiée par l'institut DIW (Berlin) et consacrée au revenu net disponible par unité de consommation (Goebel et al., 2010),

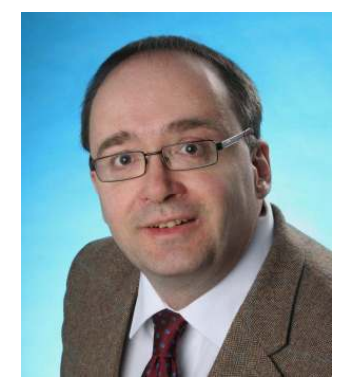

Matthias Bittorf, politologue, chercheur à la KfW-Bankengruppe, Francfort/Main

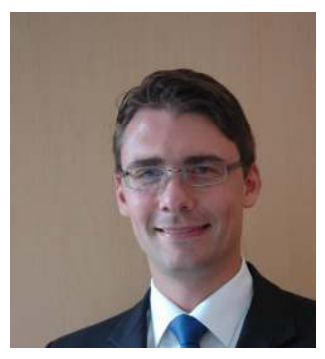

Alexander Klein, économiste, chercheur à la KfW-Bankengruppe, Francfort/Main
Inégalité en hausse depuis les années 1990 
le coefficient de Gini est passé de 0,25 au début des années 1990 à 0,29 à la fin des années 2010. L'OCDE parvient à un constat similaire : le coefficient de Gini passe de 0,25 en 1985 à 0,295 à la fin des années 2010 en Allemagne ; il reste toutefois inférieur à la moyenne de 0,314 des pays de I'OCDE (OCDE, 2011). Le coefficient de Gini mesure l'écart des revenus entre leur égalité parfaite et la situation observée ; l'échelle va de la valeur « 0 » (égalité totale) à « 1 » (inégalité maximale).

La définition du concept de revenu net disponible par unité de consommation, tel que l'utilisent par exemple l'institut DIW de Berlin ou l'OCDE (de même que l'Insee) pour calculer la répartition des revenus et les niveaux de vie, permet de comparer entre eux les ménages de taille et de composition différente.

Pour ce faire, on ne divise pas le revenu net disponible total d'un ménage donné par le nombre des personnes qui le composent, mais par la somme des unités de consommation. La pondération est alors la suivante : le chef de famille ou personne de référence équivaut à une unité de consommation, chacune des autres personnes de 14 ans ou plus à 0,5 seulement, et les enfants, à 0,3 . Cela permet de prendre en compte le fait que les personnes vivant dans un même ménage ont un avantage sur les célibataires (ou singles) en ce qui concerne les coûts fixes : il leur est plus aisé d'atteindre un niveau de vie comparable.

Ce mode de calcul part donc du principe que les ménages (personnes) isolé(e)s ont des besoins supérieurs. Or comme il présuppose notamment l'existence d'une claire tendance à la disparité des revenus, et que c'est sur ce présupposé que reposent les débats sur la pauvreté, il est fortement controversé. L'institut ifo de Munich compte parmi ses plus ardents détracteurs ; il reproche en effet à ce mode de calcul de "rompre avec le principe fondamental des statistiques, qui exige de séparer clairement les faits et les jugements de valeur » (Sinn, 2008).

Hausse brutale des inégalités à partir de $2005, \ldots$
... trop hâtivement imputées aux réformes de l'Agenda 2010
Quand on répartit en quatre périodes les années 1992-2009, on s'aperçoit que le coefficient de Gini suit une progression continue, mais qu'il enregistre une hausse brutale durant la dernière période, c'est-à-dire à partir de 2005 . Ce constat incite certains à penser que les réformes sociales de l'Agenda 2010, votées à partir de 2003 et mises en œuvre entre 2003 et 2005, pourraient s'être traduites par une hausse des inégalités en ce qui concerne les revenus disponibles.

Tableau 1 : Inégalité des revenus ${ }^{1)}$ et redistribution par l'Etat social (Gini)

\begin{tabular}{|cccc|}
\hline Total & $\begin{array}{c}\text { Revenu imposable }^{2)} \\
\text { (l'année précédente) }\end{array}$ & $\begin{array}{c}\text { Revenu net }^{3} \text { ) } \\
\text { (l'année précédente) }\end{array}$ & $\begin{array}{c}\text { Redistribution }^{4)} \\
\text { (''année précédente) }\end{array}$ \\
$\mathbf{1 9 9 2}-\mathbf{1 9 9 4}$ & & & \\
$\mathbf{1 9 9 5}-\mathbf{1 9 9 9}$ & 0,41 & 0,25 & $38 \%$ \\
$\mathbf{2 0 0 0}-\mathbf{2 0 0 4}$ & 0,44 & 0,26 & $42 \%$ \\
$\mathbf{2 0 0 5 - 2 0 0 9}$ & 0,46 & 0,27 & $42 \%$ \\
Ouest & 0,48 & 0,29 & $40 \%$ \\
$\mathbf{1 9 9 2}-\mathbf{1 9 9 4}$ & & & \\
$\mathbf{1 9 9 5}-\mathbf{1 9 9 9}$ & 0,40 & 0,25 & $37 \%$ \\
$\mathbf{2 0 0 0}-\mathbf{2 0 0 4}$ & 0,43 & 0,26 & $39 \%$ \\
$\mathbf{2 0 0 5}-\mathbf{2 0 0 9}$ & 0,45 & 0,27 & $39 \%$ \\
Est & 0,47 & 0,29 & $37 \%$ \\
$\mathbf{1 9 9 2}-\mathbf{1 9 9 4}$ & & & \\
$\mathbf{1 9 9 5}-\mathbf{1 9 9 9}$ & 0,40 & 0,21 & $48 \%$ \\
$\mathbf{2 0 0 0}-\mathbf{2 0 0 4}$ & 0,47 & 0,21 & $55 \%$ \\
$\mathbf{2 0 0 5}-\mathbf{2 0 0 9}$ & 0,51 & 0,22 & $56 \%$ \\
\hline
\end{tabular}

Source: SOEP v26; calculs de l'institut DIW de Berlin. 1) Inégalité mesurée par le coefficient de Gini $(0=$ distribution totalement égale, 1 = distribution totalement inégale). 2) Revenu imposable (revenu issu du travail et du patrimoine), pondéré selon l'échelle révisée de l'OCDE. 3) Revenu disponible du ménage (après impôts directs et transferts sociaux), pondéré selon l'échelle révisée de l'OCDE. 4) Redistribution : part de la réduction des inégalités de revenus du fait de la politique sociale (impôts directs et transferts).

Ces réformes avaient consisté notamment en la création du régime d'aide forfaitaire "Hartz IV », la réduction de la durée de versement des allocations chômage, l'obligation faite au chômeur d'accepter tout emploi considéré comme acceptable, la prise en considération du patrimoine ou des droits à pension de retraite dans le calcul des transferts sociaux, diverses mesures d'incitation au 
retour en emploi, ainsi que dans un train de mesures en matière de politique familiale, d'éducation/formation et d'assurance vieillesse. Une réforme en profondeur du système de protection sociale était devenue impérative après que le nombre de chômeurs avait dépassé le seuil des 4 millions en 2002 et que les coûts de la protection chômage grevaient de plus en plus lourdement les budgets publics. L'Agenda 2010 avait soulevé une vive controverse dans l'espace public, ses opposants estimant que l'abaissement du montant des aides aux demandeurs d'emploi décidé dans ce cadre revenait à un durcissement inacceptable des conditions ouvrant droit à prestation, et que cette réforme menait au « démantèlement de l'Etat social ». Avec le recul, les réformes de l'Agenda 2010 sont considérées comme une des principales raisons expliquant le regain de compétitivité de l'économie allemande au cours de ces dernières années.

Pour la période de la crise de la finance mondiale et de la récession, les premiers calculs effectués par l'institut DIW semblent indiquer que la polarisation des revenus ne s'est pas accrue. Après avoir atteint son point culminant en 2006, elle n'a varié depuis que de manière insignifiante, et ce même durant l'année de récession 2009 (voir Goebel et al., 2010). L'évolution favorable constatée à partir de 2007/08 s'explique notamment par la politique de soutien à la conjoncture menée par le gouvernement fédéral. Des mesures comme celles en faveur du chômage partiel (voir dans ce numéro la contribution de Spitznagel/Wanger), mais aussi celles garantissant le crédit bancaire aux entreprises, avaient largement contribué à stabiliser le marché de l'emploi.

L'écart de la fourchette des revenus - entre d'une part les revenus moyens et hauts, d'autre part les faibles revenus - s'observe aisément grâce à l'évolution des revenus mensuels. On constate ainsi que, de 1999 à 2009, les ménages aux revenus inférieurs à $70 \%$ du revenu médian ont vu leur revenu réel baisser, en valeur absolue comme en valeur relative. Ainsi, le revenu réel net pondéré par tête (unité de consommation) des ménages à faibles revenus se situait en moyenne à $685 €$ par mois en 1999, mais est tombé à $677 €$ dix ans plus tard $(-1,2 \%)$. A l'inverse, dans la tranche supérieure (ménages aux revenus supérieurs à $150 \%$ du revenu médian), on observe une hausse de $10 \%$. Quant à celle des tranches moyennes, elle a été de 3,2 \% (voir tableau 2).
La crise n'a pas accru l'inégalité

La fourchette des revenus s'écarte depuis longtemps

Tableau 2 : Une classe moyenne qui fond et un écart salarial qui s'accroît - Données clés sur la répartition des revenus en Allemagne -

\begin{tabular}{|c|c|c|c|}
\hline & \multicolumn{3}{|c|}{ Ménages aux revenus de $\ldots \%$ du revenu médian ${ }^{1)}$} \\
\hline & $\begin{array}{l}\text { moins de } 70 \% \\
\text { (faibles revenus) }\end{array}$ & $\begin{array}{c}\text { de } 70 \text { à } 150 \% \\
\text { (revenus moyens) }\end{array}$ & $\begin{array}{l}\text { plus de } 150 \% \\
\text { (hauts revenus) }\end{array}$ \\
\hline \multicolumn{4}{|c|}{ Part dans le total des ménages en \% } \\
\hline 1999 & 17,7 & 64,3 & 18,0 \\
\hline 2009 & 22,5 & 58,7 & 18,8 \\
\hline $\begin{array}{l}\text { Variation } \\
\text { (en points de pourcentage) }\end{array}$ & 4,9 & $-5,7$ & 0,8 \\
\hline \multicolumn{4}{|c|}{ Moyenne dans chaque tranche (revenu mensuel net pondéré par tête) en $€^{2)}$} \\
\hline 1999 & 685 & 1.270 & 2.436 \\
\hline 2009 & 677 & 1.311 & 2.672 \\
\hline $\begin{array}{l}\text { Variation } \\
\text { (en points de pourcentage) }\end{array}$ & $-1,2$ & 3,2 & 9,7 \\
\hline \multicolumn{4}{|c|}{ Moyenne des différentes tranches de revenus en \% du revenu de la tranche moyenne } \\
\hline 1999 & 53,9 & 100,0 & 191,8 \\
\hline 2009 & 51,6 & 100,0 & 203,8 \\
\hline $\begin{array}{l}\text { Variation } \\
\text { (en points de pourcentage) }\end{array}$ & $-2,3$ & l & 12,0 \\
\hline
\end{tabular}

Source des données : DIW, calculs de KfW Research. 1) Pour prendre en compte l'évolution du pouvoir d'achat, les revenus sont établis sur la base des prix 2005. Les données de ce tableau sont établies à partir du revenu net par tête des ménages. 2) Sur la pondération par unité de consommation, voir l'encadré supra. 
Hausse de la disparité des revenus imposables

Une politique de redistribution qui lisse les inégalités

Principale cause des inégalités : la hausse de la demande de main-d'œuvre qualifiéé

Clé de la disparité des revenus : l'accès à la formation

Le revenu s'élève avec le niveau de formation
La disparité des revenus imposables s'est accrue elle aussi. Par revenus imposables on entend les revenus bruts issus du travail (activité salariée ou travail indépendant), du patrimoine et des transferts familiaux (aide des parents aux enfants par exemple), c'est-à-dire les revenus avant redistribution au sein du système fiscal et de transferts sociaux. La hausse des inégalités est particulièrement nette entre 1992 et 1999 ; elle s'explique principalement par la différenciation qui s'est effectuée dans la structure auparavant très nivelée des revenus dans l'ex-RDA. En effet, dans la RDA de la fin des années 1980, les salaires nets des diplômés de l'enseignement supérieur n'étaient supérieurs que de $15 \%$ à ceux des ouvriers dans la production, alors qu'en RFA, l'écart pouvait aller jusqu'à 70 \% (Stephan/Wiedemann, 1990).

Aujourd'hui, dans l'ensemble de l'Allemagne, la disparité observée parmi les revenus nets disponibles par unité de consommation, c'est-à-dire les revenus après transferts sociaux, est inférieure d'environ $40 \%$ à celle des revenus imposables correspondants (voir tableau 1). Ce constat reflète la volonté d'une politique systématique de redistribution. D'une manière générale, l'Allemagne fait partie de ces pays de l'OCDE où l'inégalité des revenus est très sensiblement lissée par les transferts sociaux (soutien pécuniaire aux bénéficiaires de "Hartz IV ", complément versé à leurs enfants, allocations enfant, aides au logement) et le régime fiscal. Ainsi, $10 \%$ seulement des foyers fiscaux contribuent à hauteur de 53 \% à l'encours de l'impôt sur le revenu, et un quart des contribuables sont faiblement assujettis, voire exonérés (cf. Franz, 2010).

Le constat d'un accroissement des disparités qui s'observe depuis un certain temps déjà, et notamment celui du recul de la tranche inférieure dans l'échelle des revenus, se trouve corroboré par une récente étude menée par l'institut DIW, qui intègre l'année 2010 ainsi que le premier trimestre 2011 (Wagner/ Brenke, 2011). Les auteurs imputent cette tendance lourde principalement au recul de la demande de main-d'œuvre pour des emplois comparativement peu qualifiés et des tâches routinières. L'exemple le plus parlant est celui des retraits et versements en liquide qui ne s'effectuent plus manuellement mais sont confiées à des distributeurs automatiques. Cette évolution a pour corollaire la hausse progressive de la demande de main-d'œuvre hautement qualifiée.

\section{Qualifications et revenus}

L'accès à la formation est un facteur clé agissant sur la distribution des revenus. En Allemagne, et dans ce contexte, il s'agit le plus généralement du premier diplôme sanctionnant une formation professionnelle initiale achevée dans le système dual. Et on y part généralement du principe que les actifs disposant de la meilleure qualification formalisée sont ceux qui peuvent prétendre aux revenus les plus élevés, étant donné qu'en règle générale, leur productivité est particulièrement forte, ce qui justifie un niveau supérieur de rémunération. Cet état de fait trouve son expression dans la structure des revenus des actifs occupés selon leur niveau de formation scolaire ou professionnelle.

Le graphique ci-après présente les principaux niveaux de revenus comparés selon le niveau de formation/qualification obtenu. Ces positions respectives reflètent la relation entre d'une part le niveau médian des revenus bruts mensuels du groupe d'actifs occupés disposant, au sein de ce groupe, du diplôme le plus élevé sanctionnant une formation professionnelle initiale et, d'autre part, le niveau médian des revenus bruts mensuels de tous les actifs occupés. Ces positions sont rapportées au niveau comparé des salaires bruts horaires propres à chacun de ces groupes.

Le graphique révèle ainsi que le revenu médian d'un diplômé de l'enseignement supérieur se situe à $79 \%$ au-dessus du revenu médian de tous les actifs occupés, mais que celui d'un qualifié de l'enseignement secondaire ou d'une personne ayant achevé une formation professionnelle initiale ne dépasse ce 
dernier que de $34 \%$. Enfin, le revenu médian d'une personne disposant d'un baccalauréat et d'une formation professionnelle se situe à $64 \%$ au-dessus du revenu médian de tous les actifs occupés.

Position relative des revenus des actifs de 30 à 60 ans selon leur niveau de qualification ou de formation en 2008

(en \% du salaire brut horaire médian de tous les actifs occupés)

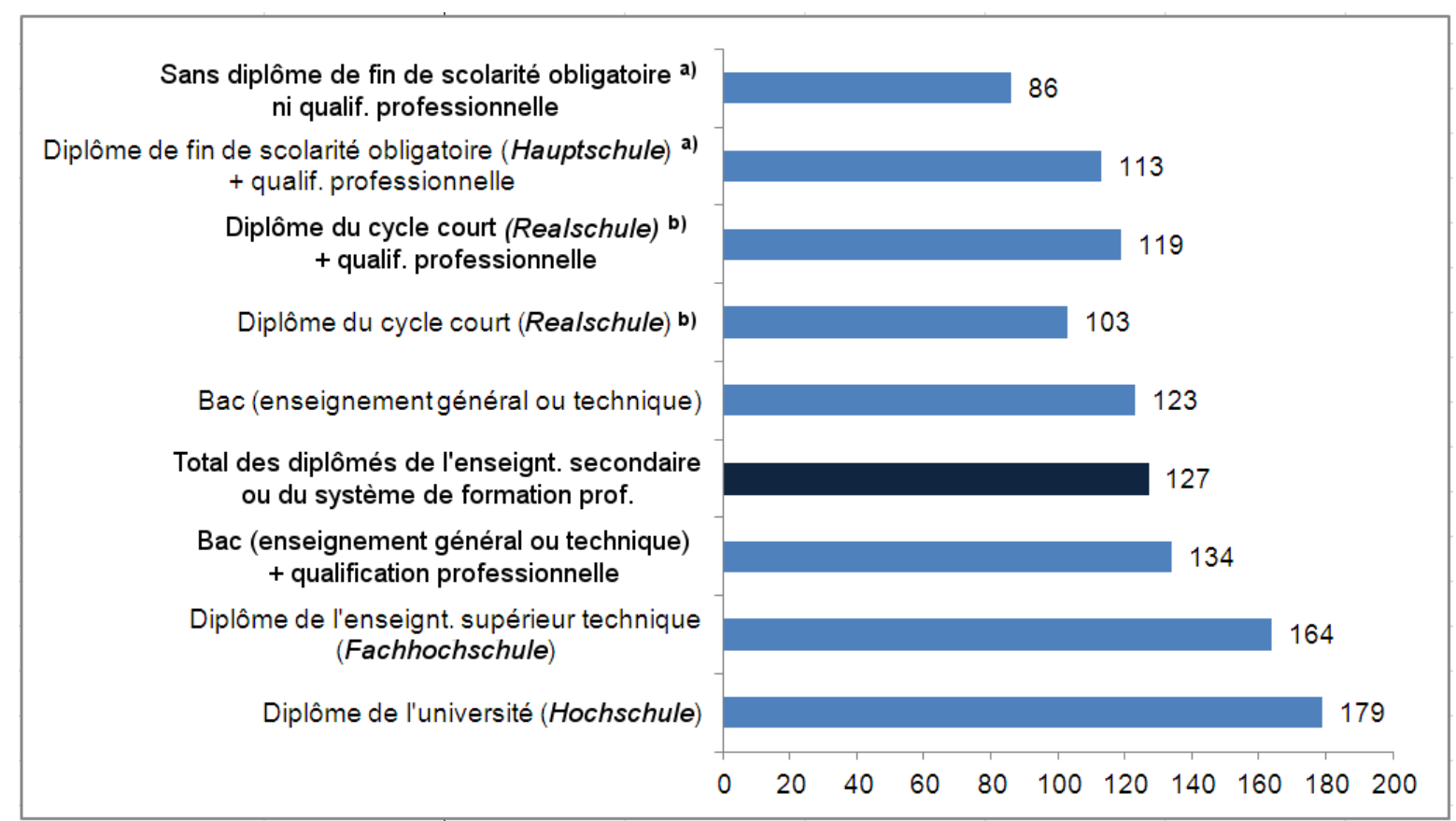

Source : SOEP 2008, calculs du DIW / Autorengruppe Bildungsberichterstattung (2010). *) Classification CASMIN. a) Hauptschule : scolarité obligatoire ( 5 ans d'enseignement secondaire) ; b) Realschule : 6 ans d'enseignement secondaire.

Ce graphique montre également que le revenu médian d'un diplômé de l'université est supérieur de quelque $58 \%$ à celui d'un actif formé dans le système dual ou d'un sortant du cycle court de l'enseignement secondaire (Realschule; voir REA 75/2006). Pour calculer cet écart, il suffit de rapporter le revenu médian des actifs diplômés du supérieur au revenu médian des actifs ayant achevé une formation professionnelle : $(179-113) / 113=0,58$, soit $58 \%$. Cet écart proportionnel (de $58 \%$ en l'occurrence) est aussi appelé le rendement de la formation (Bildungsrendite) universitaire en comparaison d'une formation professionnelle initiale. On pourrait établir de la même manière ce rendement de la formation, c'est-à-dire l'accroissement respectif des revenus, par exemple par rapport à un diplôme de niveau inférieur.

Le même constat s'observe dans les autres pays de l'OCDE : dans la moitié de ceux-ci (17 sur 32), le rendement d'un diplôme du tertiaire en termes de revenu est supérieur de plus de 50 \% (OCDE, Education at a Glance 2011).

Dans la dernière édition de son échantillon de biographies intégrées sur le marché de l'emploi (Stichprobe der Integrierten Arbeitsmarktbiographien - SIAB), l'institut $I A B$ de Nuremberg confirme le lien entre niveau des qualifications et des revenus sur le long terme (en l'occurrence 1984-2008). C'est entre les diplômés du supérieur et les actifs faiblement qualifiés que l'écart est le plus important.

Or si la hiérarchie entre les revenus en fonction des qualifications est restée stable au cours de cette période, l'écart entre les salaires bruts réels des actifs hautement et faiblement qualifiés s'est, lui, creusé. Ainsi, le surcroît salarial enregistré par les actifs disposant seulement d'un Abitur par rapport aux moins qualifiés s'est accru de 37 points, passant de $44 \%$ à $81 \%$. Quant à celui des
Rendement supérieur d'un diplôme du tertiaire

En Allemagne, la hiérarchie des revenus en fonction des qualifications est stable depuis 30 ans

Mais l'écart entre les deux extrêmes se creuse 
Haut risque de chômage pour les moins qualifiés

Diplôme du supérieur : une 'garantie d'emploi'

Baisse du taux des 'doublement qualifiés'

Déterminisme social de l'accès au supérieur diplômés du supérieur, il a augmenté de 46 points pour s'établir à $158 \%$. Dans le même temps, les salaires réels des actifs faiblement qualifiés ont renoué en 2008 avec leur faible niveau des années 1980 (Möller, 2011).

\section{Niveau de qualification, origine sociale et chômage}

En Allemagne, non seulement les actifs diplômés du tertiaire ont des revenus parmi les plus élevés, mais la probabilité d'entrer en chômage est nettement plus faible pour eux que pour les actifs peu qualifiés. En 2009, le taux de chômage n'était que de $2,5 \%$ dans la première catégorie, alors qu'il se situait à $6,6 \%$ chez ceux qui avaient achevé une formation profesionnelle initiale. A l'inverse, il atteignait 21,9 \% chez les actifs non diplômés ou sans formation professionnelle (Bundesagentur für Arbeit, 2011).

Au fil de l'histoire, le taux de chômage des diplômés du supérieur n'a certes cessé de varier quelque peu, mais il est toujours resté inférieur à celui observé chez les actifs ayant achevé une formation professionnelle. Alors qu'en 1990, les taux de chômage dans ces deux groupes étaient assez proches (respectivement de $3,5 \%$ et $4,0 \%$ ), l'écart s'est accru de 5,9 points en 2004 , avant de se réduire à 4,1 points en 2009 (statistiques de l'institut IAB sur le taux de chômage par niveaux de qualification). Autrement dit, sous l'angle de la garantie de l'emploi, les diplômes du supérieur tendent depuis quelques années à prendre le pas sur une qualification professionnelle achevée.

En Allemagne, actuellement, $23 \%$ des primo-inscrits dans un établissement d'enseignement supérieur ont achevé au préalable une formation professionnelle (BMBF, 2010). Mais ce taux est en baisse depuis 1994, ce qui s'explique par la forte diminution du nombre des actifs " doublement qualifiés », c'est-àdire de ceux qui disposent d'un baccalauréat et d'un diplôme sanctionnant une formation professionnelle achevée. Plus généralement, les cas de ceux qui entament des études supérieures après avoir achevé une formation professionnelle, surtout dans l'artisanat, demeurent rares. Il faut se garder d'en conclure hâtivement à une crise de l'apprentissage ; il reste la voie royale (voir REA 103/ 2011). Cela étant, malgré les nombreuses passerelles existantes, la formation duale et la formation universitaire demeurent deux systèmes très différents et généralement indépendants l'un de l'autre.

Plus le niveau des qualifications est élevé, plus les revenus espérés sont donc élevés eux aussi, et plus le risque de chômage est faible. Cela amène à se demander pourquoi, en Allemagne, si peu d'élèves ou de bacheliers optent pour des études dans le supérieur. Quand on regarde les données, on s'aperçoit alors que le taux d'inscription à l'université des bacheliers issus d'une famille d'ouvriers n'est que de $17 \%$ alors qu'il atteint $67 \%$ parmi ceux qui sont issus d'une famille de fonctionnaires. Ceux parmi les bacheliers dont le père est diplômé du supérieur ont comparativement une nette propension à suivre la même voie. Ainsi, $84 \%$ des enfants de fonctionnaires et $64 \%$ des enfants d'employés ayant ce niveau de qualification s'inscrivent dans un établissement d'enseignement supérieur (données 2007).

Autrement dit : le choix de suivre des études supérieures ne dépend pas seulement des capacités individuelles des jeunes Allemands, mais avant tout du diplôme le plus élevé de leurs parents, comme de la position qu'ils occupent dans la vie professionnelle. Ces deux critères sont les éléments clés du concept de " contexte socio-économique » tel qu'il prévaut dans « l'enquête sociale » (Sozialerhebung) qu'effectue à intervalles réguliers le Deutsches Studentenwerk, l'équivalent allemand du CROUS français ; mais il reste délicat à opérationnaliser (voir à ce propos BMBF, 2010).

En effet, pour définir ce « contexte socio-économique », il faudrait connaître toute une série de facteurs comme le niveau de formation ou la catégorie CSP 
des parents, mais aussi des grands-parents, le patrimoine, le lieu d'habitation, etc. Mais de telles données sont quasi impossibles à obtenir de la part des étudiants. II faut donc se contenter d'approximations, en l'occurrence d'informations sur le niveau de formation et la position professionnelle du père. Malgré cette imprécision, cette enquête n'en permet pas moins de parvenir à des conclusions probantes, et s'est donc établie comme la référence.

Reste la question des origines géographiques (ce que les statisticiens appellent le « contexte migratoire » : Migrationshintergrund) ; ces données sont recueillies indépendamment de l'enquête sociale du deutsches Studentenwerk. Bien qu'on sache ainsi que les personnes issues de l'immigration ont un statut socioéconomique de niveau souvent bas (niveau de formation et catégorie CSP inférieure du père ; voir par exemple Wahl, 2008), cet état de fait n'est jamais discuté dans le contexte du débat sur le lien entre un contexte socio-économique faible et les faibles perspectives d'évolution dans l'échelle des revenus.

Au total, on s'aperçoit donc que la réussite dans le système de formation ne dépend pas seulement de la qualification formelle (en l'occurrence l'obtention du baccalauréat), mais bien plus encore du contexte socio-économique des jeunes Allemands. C'est là aussi le constat des Etudes PISA de l'OCDE qui cherchent à permettre la comparaison internationale des qualifications des jeunes en âge scolaire. Aucun des pays de l'OCDE ne parvient à déconnecter le niveau des compétences atteint par les élèves dans les divers tests et le statut socio-économique des parents. Bien que, depuis la première étude PISA en 2000 (sur l'impact qu'elle a eu en Allemagne, voir REA 66/2004), on puisse observer une amélioration sensible en la matière, il n'en demeure pas moins qu'aujourd'hui encore, "l'ampleur des disparités sociales influant sur les compétences... reste proche de la moyenne des pays de l'OCDE » (Ehmke/Jude, 2010).

Depuis 20 ans, on observe en Allemagne une hausse des inégalités en ce qui concerne la distribution des revenus imposables comme des revenus nets des ménages. Les raisons principales de cette évolution sont d'une part la plus forte valorisation de la main-d'œuvre qualifiée dans le fil des mutations technologiques et, de l'autre, l'accroissement de la concurrence mondiale, qui ont pesé tout particulièrement sur les catégories inférieures dans l'échelle des salaires. Certes, le régime fiscal et le système de transferts sociaux ont permis de lisser considérablement une grande partie des écarts.

Néanmoins, la persistance des inégalités de revenus incite à considérer cette problématique comme un défi politique majeur - tout particulièrement pour préserver la cohésion sociale.

L'objectif crucial est dès lors l'accès à une meilleure formation de ces couches de la population qui pourraient tirer profit d'une qualification supérieure. Car c'est le facteur formation qui détermine largement la position de chacun sur l'échelle des revenus et qui réduit le risque de chômage - au moins autant que l'origine sociale, bien que l'état des statistiques ne permette guère actuellement de quantifier plus précisément la part d'un certain déterminisme social dans ces inégalités de revenus.

II n'en reste pas moins que le lien entre la réussite des jeunes dans le système scolaire et leur origine sociale est indéniable. Les bacheliers issus d'une famille d'ouvriers sont nettement moins nombreux à s'inscrire à l'université que ceux issus d'une famille d'employés ou de fonctionnaires.

Depuis le choc que suscita en Allemagne la première étude PISA en 2000, on ne compte plus les réformes engagées pour remédier à cette situation. La réussite du parcours de formation restant toujours étroitement liée à l'origine sociale des jeunes, il convient d'accroître l'équité des chances et d'ouvrir à un plus grand nombre l'accès à des formations de qualité et de niveau élevé. Ce n'est
Un constat qui vaut pour tous les pays de l'OCDE 
qu'ainsi qu'on parviendra à réduire les disparités et à préserver à terme le lien social.

(Traduction : I. Bourgeois)

\section{Indications bibliographiques}

-Bittorf M., KLein A., "Einkommensverteilung und Bildung », KfW-Research, Akzente, n 56, janvier 2012

- BMBF, Die wirtschaftliche und soziale Lage der Studierenden in der Bundesrepublik Deutschland 2009 - 19. Sozialerhebung des Deutschen Studentenwerks durchgeführt durch HIS HochschulInformations-System, Bonn, Berlin, 2010

-BouRgeols I., "Décryptage : Pauvreté ? Non, mais la classe moyenne fond", et "Pouvoir d'achat et salaires nets stagnent depuis 20 ans ?! », Regards sur l'économie allemande, $\mathrm{n}^{\text {os }} 87 / 2008$ et $83 / 2007$

- BundesagentuR füR ARBEIT, Der Arbeitsmarkt für Akademikerinnen und Akademiker in Deutschland, Nuremberg, 2011

-EHMke T., JUde N., «Soziale Herkunft und Kompetenzerwerb », in KLIEME E. (ed), PISA 2009, Bilanz nach einem Jahrzehnt, Münster/New York/Munich/Berlin, 2010

-Franz W., « Schieflagen », ZEWnews, juillet/août 2010

-Franz W., Arbeitsmarktökonomik, $7^{\mathrm{e}}$ édition, Berlin, 2009

- Glocker D., Storck J., «Uni, Fachhochschule oder Ausbildung - welche Fächer bringen die höchsten Löhne? », DIW-Wochenbericht, n 13/2012

- Goebel J., Gornig M., HäußeRmann H., «Polarisierung der Einkommen: Die Mittelschicht verliert », DIW-Wochenbericht, $\mathrm{n}^{\circ}$ 24/2010

-KöCHER R., « Produzieren wir eine Schicht sozialer Verlierer ? », Frankfurter Allgemeine Zeitung, 17-08-2011

- Mendolicchio C., Rhein T., « Wo sich Bildung für Frauen mehr lohnt als für Männer », IAB-Kurzbericht, $n^{\circ} 5 / 2012$

-MöLLER J., «Qualifikationsbedingte Lohnunterschiede - Wer kriegt wie viel Butter aufs Brot ? », IAB-Forum, $\mathrm{n}^{\circ} 1 / 2011$

-OCDE, Divided We Stand - Why Inequality Keeps Rising, Paris, 2011

- OCDE, Education at a Glance 2011, Bildung auf einen Blick 2011, OECD-Indikatoren

- SinN H.-W., « Der bedarfsgewichtete Käse und die neue Armut », ifo Schnelldienst, n 10/2008

- «SPD-Chef sorgt sich um wachsende Unterschicht», Spiegel Online, 07-06-2010 (http://www. spiegel.de/politik/deutschland/0,1518,441376,00.html)

- Stephan H., WiedemanN E., « Lohnstruktur und Lohndifferenzierung in der DDR, Ergebnisse der Lohndatenerfassung vom September $1988 »$, Mitteilungen aus der Arbeitsmarkt- und Berufsforschung, $n^{\circ} 4 / 1990$

-WAGNER G. G., BRENKE K., « Lohnpolitik. „Nötig sind kräftige Lohnerhöhungen“ », Berliner Zeitung, 05-08-2011

-WAHL S., "Les vraies causes de la disparité des revenus en RFA », Regards sur l'économie allemande, $\mathrm{n}^{\circ} 88 / 2008$. 